\title{
Virtual Reality Applications for Higher Educations: A Market Analysis
}

\author{
Jaziar Radianti and Tim A. Majchrzak \\ University of Agder, \\ Kristiansand, Norway \\ \{jaziar.radianti,timam\}@uia.no
}

\author{
Jennifer Fromm and Stefan Stieglitz \\ University of Duisburg-Essen, \\ Duisburg, Germany \\ \{jennifer.fromm,stefan.stieglitz\}@uni-due.de
}

\author{
Jan vom Brocke \\ University of Liechtenstein, \\ Vaduz, Liechtenstein \\ jan.vom.brocke@uni.li
}

\begin{abstract}
Benefits and applications of virtual reality (VR) in higher education have seen much interest both from research and industry. While several immersive VR applications for higher education have been described, a structured analysis of such applications on the market does not exist. We use design elements from research for applying VR in higher education to analyze available $V R$ apps. The analyzed VR applications were acquired from pertinent online stores to capture the market's state. We analyze the current picture of the available apps by categorizing them based on design elements and learning content. The aims are to map what types of apps are available, to study what expected types cannot (yet) be found, to compare the current state of the literature and the educational VR app market, as well as to scrutinize the most frequently used design elements for VR in education.
\end{abstract}

\section{Introduction}

Head-Mounted Display (HMD) Virtual Reality (VR) is steadily gaining popularity $[1,2]$. It is not merely used for entertainment but also for education purposes for several reasons. VR is immersive and can bring students into realistic scenes and experiences, which may not be achievable in the real world [3]; think, e.g. of experimentation in harmful environments. The visual experience can be strengthened with audio. Moreover, VR can be designed in a very interactive way, allowing the users to touch, grab, assemble-disassemble objects, moving around in the virtual world and many other possibilities [4]. Some universities have explored and initiated "Accelerated Immersive Education" to open up the possibilities to create a global classroom curriculum, allowing students from different parts of the world to access the same learning materials [5].
Immersive VR technologies become more accessible, both considering those that require high budgets - such as Oculus Quest - and those for low budgets - such as Google Cardboard [6, 7]. However, it has not been systematically analyzed what kinds of apps are available on the market that support learning in the context of higher education. To the best of our knowledge, no comprehensive market analysis exists, yet. Therefore, we set out to close this gap. We explore the VR app market to understand the current situation of the possibility to adopt VR in teaching.

Consequently, the main contribution of this article is the first overview of the app market for immersive VR in higher education. We expect this to be a valuable resource for researchers and input to future theory building papers in the field as well as relevant to educators in practice. This article also compares the current state of the art of HMD applications in the literature and the reality of app availability in the educational VR app market.

The remainder of this article is structured as follows. In Section 2, we discuss previous studies of VR in the education app market. We then elaborate on our data sources and methodology in Section 3. Section 4 presents our frameworks for analysis. The results from our market analysis are given in Section 5 before we discuss them in Section 6. Section 7 draws a conclusion.

\section{Previous Works}

We found several systematic literature reviews of VR applications in education $[8,9,10,11,12]$. For example, Freina and Ott [8] analyzed scientific articles with regard to the advantages and drawbacks of VR use in education. Kavanagh et al. [10] instead focused on application areas and motivations of VR use in education. The systematic review of Chavez and Bayona [9] analyzed the current state of the literature with regard to design elements of educational VR apps and their 
effects on the learning process. Wohlgenannt et al. [11] focused on scientific literature about VR applications in higher education and created a systematic mapping of design elements implemented to teach different types of learning content. The most recent systematic literature review analyzed 38 scientific articles about VR applications in higher education with regard to technology type, applied learning theories and research methods, application domain, learning content, and design elements [12]. The authors provide a systematic mapping of fourteen design elements implemented to teach different types of learning content [12]. Both, Wohlgenannt et al. [11] and Radianti et al. [12], suggest to extend their work by conducting a comprehensive market analysis of educational VR apps.

Our literature review revealed that there is indeed a research gap in this regard. We found several analyses of mobile app markets with a focus on education; however, there was no specific focus on VR apps [13, 14, 15, 16]. For example, Shuler [13] analyzed the 100 top-selling paid apps in the education section of the iTunes App Store with regard to target age, subject, and price. Hayes [14] collected 1076 educational mobile apps listed on Graphite.org - a website to help educators find educational digital resources. The author focuses on the potential of mobile apps to support 21st century skills such as creativity, critical thinking, communication, and collaboration. Penchenkina [15] identified 117 mobile apps affiliated with Australian universities in the Google Play Store and proposed a typology of mobile apps in higher education based on their primary purpose, secondary purpose, and design elements. The most recent market analysis analyzed the top 10 premium and top 10 free math and literacy preschool apps from the Apple App Store, Google Play Store, and Amazon with regard to their educational features [16].

A search for market analyses focusing on both VR as a technology and education as an application domain - yielded only scarce results $[17,18]$. Stojšić et al. $[17,18]$ present 16 Google Cardboard VR apps and describe how these can be used in geography education. Karsli and Karsli [18] searched the Steam Store, Google Play Store, Apple App Store, and the Oculus Store for VR apps that were developed for language learning. The authors identified 15 apps and examined these in terms of their general features, the language skills they aim to develop, and the language learning activities [18]. It becomes apparent that current market analyses of VR apps in education are limited to a small number of apps in a specific education domain (i.e. geography, language learning). With our study, we contribute a comprehensive market analysis of VR apps in education.

\section{Data Sources and Methodology}

In the following, we first explain how we selected the app markets included in our analysis. Then, we explain our filtering approaches for selecting apps, starting with the semi-automated and followed by the manual one. Eventually, we elaborate on our coding process.

\subsection{Selection of App Markets}

To understand the immersive VR app markets, we explored the well-known app stores, which was done until March 2019. We selected three main VR app stores, i.e., Steam ${ }^{1}$, Vive ${ }^{2}$, and Google Play ${ }^{3}$. The web crawl technique [19] was used to systematically browse the web, identify the hyperlinks in the pages and automatically add them to the list of URLs to visit each market. We used the Python package Beautiful Soup ${ }^{4}$.

For the Vive and Google Play stores, which specifically focus on games, we crawled all apps available by March 2019, while for the Steam Store, we only crawled the VR apps. Note that we were aware of other VR app stores such as Oculus, iTunes Apple, and Play Station. However, these stores prohibit crawling activities (and provide no free API access which would otherwise serve our needs to identify matching apps), and hence we did not review them. We deemed existing markets to be sufficient as many VR mobile apps available in iTunes were likely to be available in Google Play. Likewise, many VR apps available in Oculus may have been covered in either Steam or Vive, or both. For example, the app Star Chart for Oculus consisting of a real-time simulation of the visible stars from the Earth, planets, and 88 star constellations is accessible in Vive and Steam. Most accessible VR apps in the Play Station store are entertainment-oriented.

We obtained 5632 apps in total. The datasets were organized in a spreadsheet, consisting of information on the source of the market, URL link to each identified app, app name, and the description of the app. To proceed with the market analysis, we conducted both semi-automatic and manual filtering processes to include and exclude the apps.

\subsection{Semi-Automatic Approach}

A two-step semi-automatic approach for inclusion and exclusion of apps was conducted. First, we ran an inclusion process that was only applied to the Steam results, as we had included all games in the crawling

\footnotetext{
${ }^{1}$ https://store.steampowered.com

${ }^{2}$ https://www.viveport.com

${ }^{3}$ https://play.google.com

${ }^{4}$ https://pypi.org/project/beautifulsoup4/
} 
process, and not the VR-apps only. The inclusion keywords were virtual reality, VR, oculus, vive and immersive. The screening process was done by developing a simple script that would go through the description text and mark the app being relevant or not relevant.

Second, the inclusion process that was valid for all apps from the three markets where we used more intuitive keywords that would fit our goal, i.e., to find education- or learning-oriented apps. Thus, we adopted the following keywords for this second semi-automatic process: learn, learning, learned, educated, education, educating, educate, train, trained, training, and trainer.

Third, we ran an exclusion process. To achieve this, we carried out a content analysis of all apps from the three markets. To understand which relevant keywords could be used for including the apps, we used the software KHCoder 3, which supports text mining and quantitative content analysis [20]. Several pre-processing activities were done, such as removing punctuation marks, stop words, and converting conjugated or inflected adjectives and verbs into their word stem and ignoring prepositions, adjectives, and adverbs. The tool helped us to extract clusters of words that frequently appeared together. The result of this process was a list of word clusters, which then were selected in a way that it allowed us to find good enough keywords for inclusion criteria.

The selections of the following keywords were adequate to exclude irrelevant apps: sairento, regatta, marine life, air combat, space bit attack, fightttris, legion, janus, sandbox, adventure game, 3D printing, hungry, cinderella, relaxation, sports, sport, soccer, jam studio, railway, horror, mix reality, baskhead training, sailing, chinese cook, fighting, and fun game. In all these three semi-automatic processes, a quick human checking was performed to ensure excluded apps were truly not relevant. The results of the crawling and semi-automatic filtering can be summarized as follows in Table 1.

Table 1. Semi-automatic checking results

\begin{tabular}{lrrrr}
\hline Stages & Steam & Vive & Google & Total \\
\hline Crawling results & 3986 & 1396 & 250 & 5632 \\
\hline Inclusion (Steam) & 3291 & & & 4937 \\
\hline Inclusion (All) & 521 & 93 & 41 & 655 \\
\hline Exclusion (All) & 370 & 161 & 41 & 572 \\
\hline
\end{tabular}

\subsection{Manual Filtering Approach}

For the next process, we merged all results into a single list consisting of $572 \mathrm{VR}$ apps. In the manual process, the authors manually read all app descriptions and excluded all apps that were not relevant. It was done by three parties, which categorized the same list as "VR app for education", "questionable", and "not relevant", and compared the results. Any discrepancies were discussed until a single option was agreed: to include or to exclude the app. This process was considered as a cross-validation process, ensuring the list was better representing what we looked for, and eliminating bias and misinterpretation. In addition, we removed duplicates, because after merging the apps from different stores, some duplicates were found. The final list ready for coding contained 120 apps, as illustrated in Table 2.

Table 2. Manual checking results

\begin{tabular}{lc}
\hline Stages & \# of Apps \\
\hline Merged list after inclusion-exclusion & $572 \mathrm{Apps}$ \\
\hline Duplication check & $477 \mathrm{Apps}$ \\
\hline List of apps ready to code & $120 \mathrm{Apps}$ \\
\hline
\end{tabular}

\subsection{Coding Process}

The coding process was carried out to classify the apps into meaningful categories. A series of frameworks organized as a concept matrix [21] has been prepared to categorize the apps. Our goal was to identify the following: the application areas, the design elements, and the learning contents, as presented in Section 4. The coding process was done by two co-authors who went through the app descriptions and each app URL in our list, observed the comments from the users of the apps, and watched the video trailer of each app. These two persons who went through all the apps also were a way for ensuring intercoder-reliability. We found some discrepancies on interpreting the categories, and we solved it through a discussion with all co-authors until agreed categories were achieved.

\section{Framework}

We applied and extended the frameworks for VR design elements and learning contents proposed by Radianti et al. [12], which were developed to understand the design elements and learning contents of VR that have been proposed in the literature. As argued in Section 2, it is the most recent and at the same time most comprehensive paper proposing such a 
classification scheme. However, in this work, we did some modifications, expanded some definitions, and renamed some categories based on the new elements discovered in the app market. These cater for the differences between scientific literature and situation on the app markets, as well as to changes in the rapidly evolving field of VR in education. The modification of the definitions are signified with italic text.

\subsection{Design Element Framework}

Realistic surroundings: The virtual environment is of high graphic quality and has been designed to replicate a specific environment in the real world.

Passive observation: Students can look around the virtual environment. This design element also applies to applications in which users can travel along a pre-defined path and look around while doing so. However, they are neither able to move around on their own nor to interact with virtual objects or other users.

Moving around: Students can explore the virtual environment on their own by teleporting or flying around.

Basic interaction with objects: Students can select virtual objects and interact with them in different ways. This includes retrieving additional information about an object in written or spoken form, taking and rotating it, zooming in on objects to see more details, and changing an object's color or shape. Some apps allow using hand gestures to interact with objects.

Assemble, disassemble, and dissect objects: Students can select virtual objects and put them together, including the creation of new objects by assembling several individual objects. On the contrary, the students can also disassemble objects to learn about small parts of the objects such as machines, or dissect an animal such as a frog, to learn about its anatomy.

Interaction with other users: Students can interact with other students or teachers and attend a virtual class. The interaction can take place in the form of an avatar and via communication tools such as instant messaging or voice chat. Some designs incorporate the real-time communication feature. This design element also includes the possibility of students visiting each other's virtual learning spaces.

Role management: The VR application offers different functionalities for different roles. A distinction is made between the role of a student and the role of a teacher. For a teacher, the VR application offers extended functionalities, such as assigning and evaluating learning tasks or viewing the learning progress of students.

Screen or result sharing and virtual presentation:
The VR application allows students and teachers to stream applications and files from their local desktop onto virtual screens. This allows them to share and edit the learning content from their local desktops with other users in the virtual environment, or to present the content directly in the virtual world.

User-generated content: Students can create new content, such as 3D models, and upload this new content to the virtual environment. This design element also applies when the user-generated content can be shared with other users so that they can use it in their virtual environment as well. This design element does not apply when students can only access virtual objects that were created by developers and provided by a library in the virtual environment.

Instructions: Students have access to a tutorial or to instructions on how to use the VR application and how to perform the learning tasks. The instructions can be given by text, audio, or a virtual agent. The virtual agents can be a virtual instructor or a virtual narrator. The text instruction can be presented in a virtual dashboard, or virtual presentation tools such as $T V$, Video or projector.

Interactions with a virtual agent: Students can talk and discuss with a virtual agent when learning. It is different from the instruction design element where the virtual agents only provide instructions. This element is especially used for language learning.

Immediate feedback: Students receive immediate textual, auditory, or haptic feedback. The feedback informs students about whether they have solved the learning tasks correctly and whether interactions with virtual objects were successful.

Knowledge test, questionnaire and exercise: Students can check their learning progress through knowledge tests, quizzes, or challenges.

Virtual rewards: Students can receive virtual rewards for successfully completing learning tasks. Students can be rewarded virtually by receiving achievements, badges, higher ranks on a leader board, and by unlocking exclusive content, such as hidden rooms or additional learning content.

Making meaningful choices: Students learn in the virtual environment through participating in a scenario (role-playing) that can end in different ways. In this scenario, they have to make decisions that affect the outcome of the scenario. This design element does not apply when the students' decisions have no influence on the outcome of the scenario.

Elevated Challenges: Students can receive more advanced learning challenges, as soon as they complete a certain task.

Alternative learning venue: Students can enter different 
types of learning venues, depending on the learning needs, for example, to enter a big auditorium with a lot of audiences to train public speaking.

\subsection{Learning Content Framework}

Analytical and problem-solving: Whether the use of VR can encourage students to improve their analytical skills, such as collecting and analyzing data, writing computer programs, or making complex decisions.

Communication, collaboration, soft skills: Whether the use of VR is intended to strengthen the students' ability to work in a team or whether students can improve their communication skills (e.g., presenting in front of an audience). This category also includes soft skills, such as management and leadership competencies.

Procedural-practical knowledge: Where the use of VR aims to assist students with internalizing procedures, such as knowing how to perform a surgery or how to perform firefighting procedures.

Declarative knowledge: Where the use of VR is intended to help students memorize factual knowledge (e.g., theoretical concepts and scientific principles). This includes, for example, learning the names of planets in our solar system.

Learning a language: Where the use of VR aims to improve students' foreign language capabilities, such as reading, listening, writing, and speaking.

Behavioral impacts: Where the use of VR aims to change the behavior of students by, for example, improving their learning habits, awareness of mobbing, and compliance to rules.

\section{Results and Analysis}

Recall that this work extends Radianti et. al.' [12] literature review, but extends the proposed framework for the market analysis (as explained in Section 4). Results are linked to this work as a point to compare and to understand the gap between the findings in the literature and in the VR app markets.

\subsection{Classification Results}

When investigating the teaching areas of VR, we identified that procedural-practical and declarative knowledge development jointly occupy the maximum (and almost identical) portion of the learning outcomes in both analyses. In their literature review, Radianti et al. [12] found that $60 \%$ of the learning contents teach developing such knowledge. In contrast, our market analysis identified that a similar learning outcome was targeted by $68.4 \%$ of the examined apps.

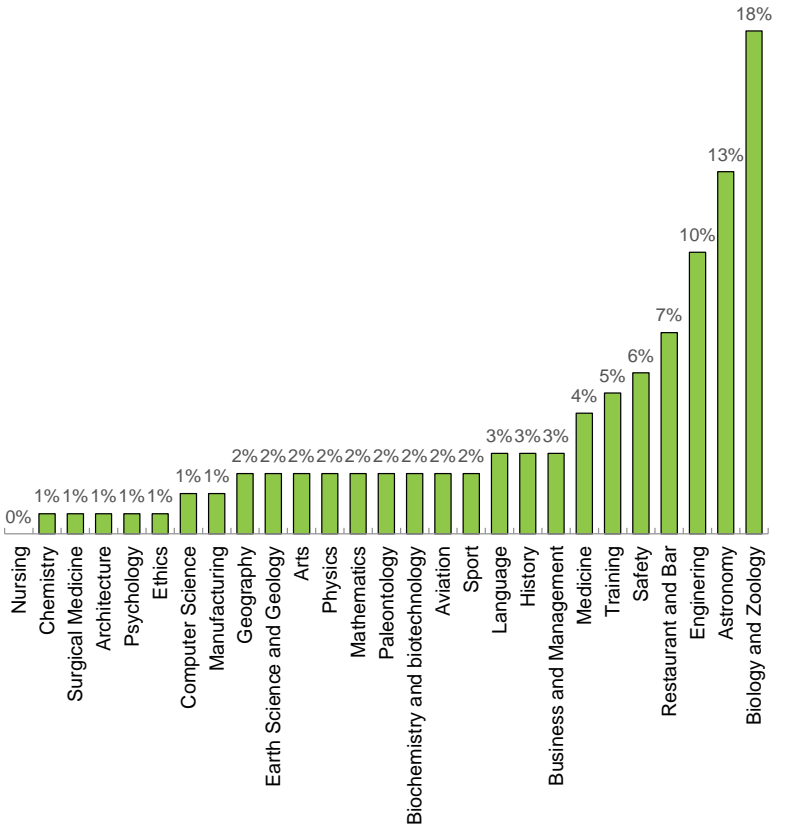

Figure 1. Application domains $(n=136)$

However, when individually analyzed, such knowledge development criteria exchange priorities in literature and market analysis. The literature review prioritized procedural-practical knowledge (34\%) development over declarative $(26 \%)$, whereas our market analysis emphasized more on declarative knowledge (43\%) development than procedural-practical (25.4\%). Other criteria kept identical ranking in both studies, although some numerical ups and downs were articulated.

In the literature review, Radianti et al. [12] summarized 17 application domains of VR that included topics from Science, Art, Safety, and Language. Along with these topics, our market analysis recognizes 10 more application domains broadly from Sport, History, Training, Business, Management, and so on. Figure 1 demonstrates the application domains identified from the market analysis. Radianti et al. [12] articulated Engineering (24\%) as the most concentrated application domain in the review, but our market analysis shows that most educational apps are developed for serving needs in the Biology and Zoology domain (18\%). In the VR app stores, the app intended for Computer Science domain is not so popular. In the study of Radianti et al. [12], $10 \%$ of the reviewed articles were from Computer Science in contrast to $1 \%$ of the apps in the app stores; Astronomy achieves greater attention in practice (from $7 \%$ to $13 \%)$.

However, regardless of the differences in terms of magnitude of similar subjects both in market analysis and literature, the application domains vary to a great 


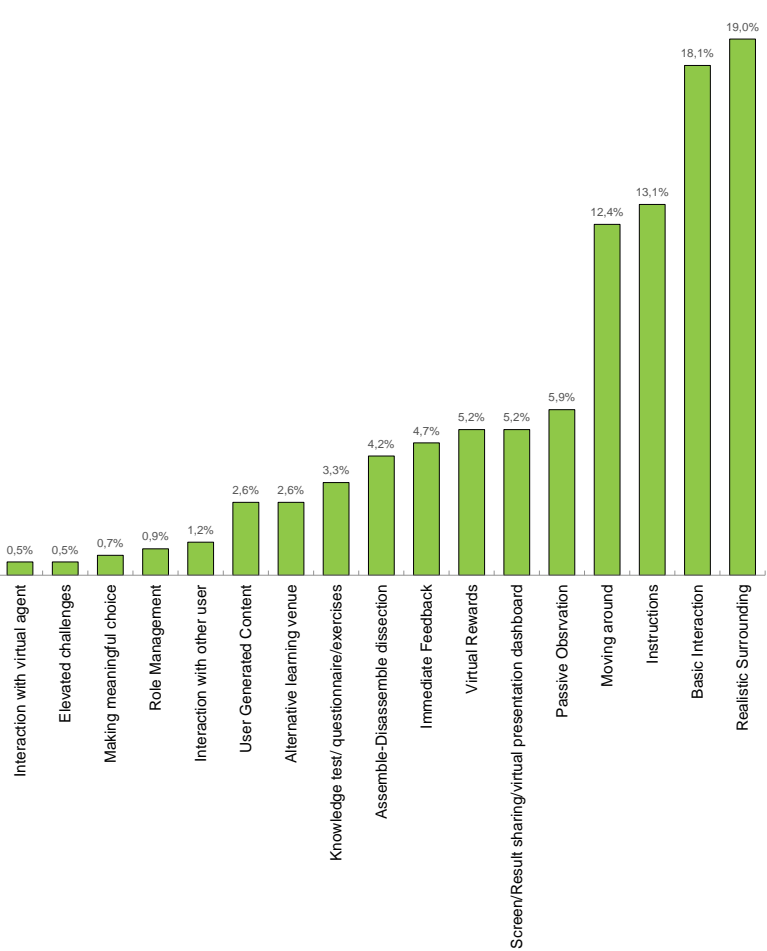

Figure 2. Design elements $(n=426))$

extent in the market. Therefore, the percentage of the application domains are more equally distributed, apart from the top five subjects. This may indicate diversities in future trends in educational VR. We notice that in our market analysis, health-related domains such as medicine and surgical medicine are lower than in the findings of Radianti et al. [12]. Moreover, no application in Nursing domain, while, the literature review [12] reported a 5\% share of this domain in the analysis.

After examining the collected apps, we identified three new design elements in addition to the ones reported by Radianti et al. [12]: interaction with virtual agents $(0.5 \%)$, elevated challenges $(0.5 \%)$, and alternative learning venues $(2.6 \%)$. The authors reported that basic interaction (24\%) and realistic surroundings $(17 \%)$, were the most frequently used VR design elements for higher education. Our analysis provided a similar outcome but prioritized realistic surroundings (19\%) over basic interaction (18.1\%). Figure 2 illustrates the overall distribution of the elements used for creating VR applications.

For effective VR support in higher education, the finding indicates that creating real-life surroundings is as vital as incorporating basic interactions with the operational environment. With a little increase in the contributing percentage, issues with instructions ranked third in both analyses. However, the market analysis prioritized enabling moving around (12.4\%) over immediate feedback (4.7\%), which was reversely reported in the literature review. Therefore, our study reveals that VR support will be more interactive if the user can move with the VR-devices rather than just receiving some immediate feedback. The significant hype of virtual rewards (from $2 \%$ to $5.2 \%$ ) and screen sharing (from $1 \%$ to $5.2 \%$ ) indicates their importance to include in VR applications for higher education.

\subsection{Mapping Results}

The mapping of learning content and design elements is visualized in Figure 3 (next page). Declarative knowledge has been employed in most of the identified learning contents of the VR apps. Elements, such as realistic surroundings, basic interaction, moving around, and instructions, need a better theoretical understanding of the subject matters for designing related VR apps. However, we have not evaluated whether these design elements actually improve procedural-practical skills. Moreover, out of the above-mentioned design elements, basic interaction, instructions, and realistic surroundings contribute to developing communication, collaboration, and soft skills. Passive observation, along with the influential design elements for previous learning content, influences behavioral impacts related learning outcomes. It is mostly observed for learning languages.

The mapping of learning content and application domains is visualized in Figure 4. Apps in Safety and Training domains are mostly tailored to develop procedural-practical knowledge, whereas apps in Biology and Zoology, Astronomy provide declarative knowledge. Although teaching analytical and problem-solving skills is essential to Engineering, it is prevalent for Safety, Mathematics, Business and Management. In theory, VR should support the important learning outcomes for different application domains, but we have no objective data about the actually needed learning outcomes in each application domain.

We also found several gaps in the markets. First, all identified apps were claimed to be designed for education, however, we could not identify what kind of learning theories have been used to guide the app development. Therefore, it was not yet completely clear, how far the VR apps available in the app stores could actually support the learning needs of the students. Second, some learning contents were only found in limited application domains, especially those that targeted behavioral impacts and language 

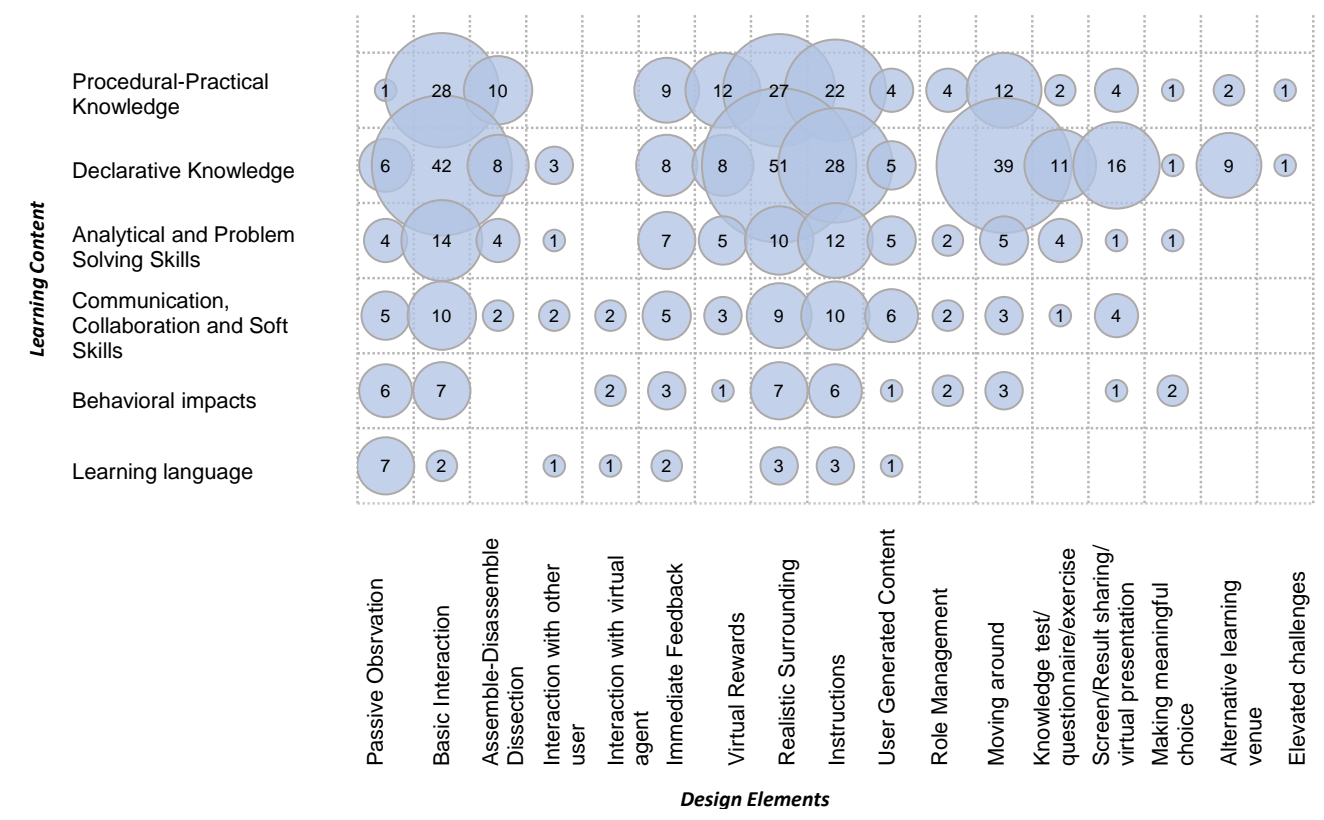

Figure 3. Mapping of learning content and design elements
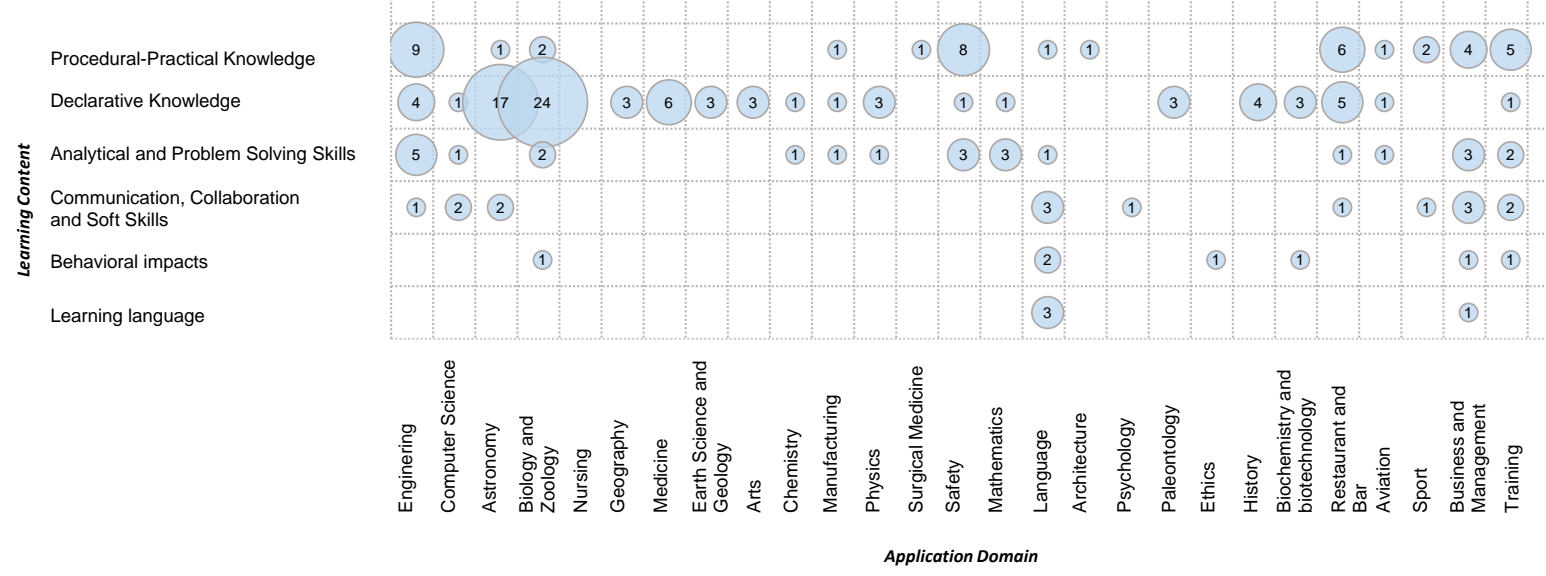

Figure 4. Mapping of learning content and application domains

learning. These two categories were identified but were not the most common reasons why VR apps were made. Perhaps, changing behaviours or learning language are still not yet areas which developers consider be meaningful. However, we found one app that focused on biology and environment, but in the spirit, the app teaches users for changing the attitude toward certain animals that are important in the food supply chain, as explained in the introductory narration of the app.

\section{Discussion}

Based on the analysis of the app market with the help of the framework, we discuss our results. First, we name lessons learned from the analysis. Second, we give recommendations for the adoption of VR apps by educators and for developers of VR apps for education. Third, we discuss limitations of our work. Fourth, we sketch directions for future research.

\subsection{Lessons Learned}

Our analysis of the market has led to several insights. First, one of the keys for successful VR apps for education is to have adequate information on the usage and benefits of the app. The majority of identified VR apps in this study adopted carefully the Instruction design element, which is apparently a key 
for a successful VR app. This design element can be manifested in various forms, ranging from virtual narration, virtual agent, virtual dashboard, and audio and text tutorials. The importance of the Instruction design element, was not so obvious in the academic literature [12].

Second, VR apps for computer science domain are not so popular in the market compared to the literature findings. It seems that such apps are frequently developed in research. Thus, Computer Science might not actually be one of the most meaningful application domains for educational VR. One of the possible explanations is that the VR apps developed in the literature can be biased by the subjects of the authors - likely researchers in this field who typically create software artifacts as part of their work anyway. From the market perspective, it is not yet so intuitive and common that people would go to the VR app stores to find e.g. how to learn coding and programming. Until now, coding skills can be improved by using other forms of digital learning tools instead of HMD VR.

Third, we have analyzed the selected 120 apps for education from the VR app stores. The educational content of these apps looks promising, but only few are really deep (i.e. comprehensive, sophisticated) enough that the learning process would not be concluded in just one session. Many reviewed apps seem to target one time use by design. Thus, they are rather supplements to enhance the understanding of students on a specific topic. This might be owed to the immaturity of the field, and to the experimental nature of many apps.

Fourth, high-quality, HMD-based VR learning materials for higher education in fact are not always offered through the VR app stores, e.g. for medicine and nursing, safety and engineering domains. Such apps use direct marketing, and they are often quite costly or require a special agreement with the potential users.

Fifth, results of our market analysis only partly align with findings from the scientific literature (cf. Sections 2 and 5). This could be explained by the novelty of the field and by the rapid pace with which it is evolving; however, it also hints to a need for additional research.

\subsection{Recommendations}

Based on the insights of this market analysis, we have the following suggestions concerning possible adoption of immersive VR in higher education, both for lecturers and for VR developers.

Educators need to be aware that the current possibilities to use VR in higher education are limited. The technology allows for abundant possibilities and examples of sophisticated apps exist; however, there is no acknowledged way how to decide on the means of virtualizing an existing course, there are no general guidelines for app usage, and there is not much evidence for effectiveness criteria for apps. All of this is owed to the immaturity of the field combined with the relatively low level of scientific coverage, which arguably is strongest regarding the technological underpinnings. At the same time, it is undeniable that VR offers the chance for profound (positive) changes to education. Our first recommendation is, thus, to embrace these possibilities, to be bold in following ideas, yet to refrain from unrealistic expectations. Although - as evidences by papers like this - there is research that tries to provide advise, adopting VR for a course will remain experimental for the near future.

Educators need to also be aware of the limited scope of apps offered in app markets. At the same time, these app markets offer many VR apps not intended for education. Our second recommendation is, thus, to be open minded. Even if existing educational apps offer limited functionality, they can serve as inspirations; VR apps built for entertainment can showcase the technology and help devising serious apps.

The third recommendation is to be patient. Obviously, the field is evolving. If educators cannot find the tools, the guidelines, or the evidence they need, it would not be right to despair. Rather, it is likely that revisiting the same VR teaching idea a year later would reveal new possibilities, maybe even an existing app. Developers need to be aware that educators might neither have a complete understanding of the technological underpinnings of their ideas nor of the progress of the technology. Our fourth recommendations is to work closely together. While this would be a common value in software engineering, putting particular emphasis on requirements engineering is advisable.

Due to the missing theory and particularly the gaps of understanding of didactic aspects of VR apps in education, developers need to be agile. If educators work exploratory and experiment with ideas, developers need to follow pace. Apps that allow tailoring, customization, frequent updates, and if need be a full redesign should be favoured, even if they come with less functionality than an app built in a more static fashion could have. Therefore, the fifth recommendations is to allow for as much change as possible.

\subsection{Limitations}

There are three kinds of limitations relevant for our work. First, there are limitations to the design and execution of our analysis. We only identified 
apps from the popular VR app stores. Moreover, we could not crawl all VR markets such as Apple's App Store and Sony's PlayStation Store, as crawling was prohibited in these two markets. Furthermore, the store markets may not offer an educational-professional app for teaching medical students such as a tool for conducting specific surgery procedures. Such apps could be commercially marketed and directly offered to the - relatively small - base of institutions that could reasonably offer them to students. However, literature has cited professional VR tools for conducting training in the medical domain [22, 23]. Possibly apps are too targeted to a specific audience, are a proprietary solution, or circulate in a limited environment. In these cases, access through the app stores may be undesirable. Examples are the Osso VR platform ${ }^{5}$ that use HMD for medical training, and XVR simulation ${ }^{6}$ for emergency management. In other words, professional VR apps for education that use direct-selling methods are not covered in our survey. The two examples are procedural-practical oriented apps. There may be more similar apps targeting the same learning contents, which are not fully captured from just surveying the VR apps in the popular markets, and indicate the opposite: the majority of accessible apps have declarative-knowledge as learning content. This study focuses on apps that use immersive VR technologies (HMDs). There may be more advanced learning apps for higher education using non-immersive technologies. It is also impossible to know the barriers from the students who use these VR apps.

Second, there are inherent limitations to the market of immersive VR apps. So far, these markets have been known as entertainment and game-oriented markets instead of markets for VR apps for education. Thus, the adoption rate of this type of app may not be as high as those that are designed for entertainment. We also did not analyze the popularity of the apps, such as how many people have downloaded the apps, what ratings apps got, and what kinds of comments have been given in reviews. Such analysis would also be tricky, since for example the amount of downloads does not necessarily relate to the number of users from higher education institutions and since ratings in app stores might be biased. In addition, we notice that advanced immersive VR for education is available but the companies would not market their products through popular app markets (which aligns with the first limitation).

Third, due to the rapid proliferation of the field, our market analysis is a first step and a snapshot. It builds on a solid framework, but it cannot yet draw from a

\footnotetext{
${ }^{5}$ https://ossovr.com/

${ }^{6}$ https://www.xvrsim.com/en/
}

profound base of theoretical work in the field.

The limitations do not lower the value of our work, particularly in the light of it being the first comprehensive market study. However, future work needs to try to address these limitations. They may also become obsolete due to the technological and content-oriented progress in the field.

\subsection{Future Research Directions}

While our recommendations target practice, the market analysis' results suggest much room for future research. One strength of VR lies in its ability to immersively engage users in the virtual environment for teaching procedural knowledge, which is in fact not so much available in the common app stores. This can be a room for improvement.

Our mapping reveals which design elements are most commonly implemented to design specific learning outcomes. However, we have little knowledge on what design elements can support learning effectively. In future research, it has to be thoroughly evaluated in experiments and real courses, which design elements are useful and actually improve intended learning outcomes. Furthermore, we require not only the technology but also new didactic concepts that provide educators with guidance on how to meaningfully integrate VR apps in their courses. Research on VR technology for education ought to be a truly interdisciplinary endeavour.

Many apps in the markets tried to "replace" teachers with virtual agents in the app. However, the efficacy of such replacement is yet unknown. It is rather a research agenda on the design elements: how far the teacher's role can be replaced, in VR-based teaching, and how to efficiently embrace VR-based teaching into the curriculum. A case study that observes the usefulness of the design elements in supporting the research contents would provide significant contributions.

Future research should try to grasp the work on VR technology for higher education in its full breadth and theorize it. While the work with learning content, design elements, and application domains is a first step, a kind of morphological box for educational VR apps could be helpful. It would support classification and allow to abstract from single fields and single approaches, allowing to understand the - likely recurring - building blocks of successful apps.

\section{Conclusion}

We have examined and explored the market for VR apps. Thereby, we gained important insights on existing VR apps and extended the VR design element framework of Radianti et al. [12]. We 
carefully examined existing apps in the popular VR app stores such as Google Play, Steam and Vive, focusing on the design elements and learning content. Our market survey identified Biology/Zoology, Astronomy and Engineering as the three most popular application domains. However, results also shows that these HMD VR apps have been developed for 27 application domains, which indicate the trends of broader adoption of VR for education. Results partly align with findings from the literature, but there is a gap between scientific work on VR for education and the reality on the app markets.

\section{Acknowledgements}

The authors are grateful for Joshua Handali who conducted the app market crawling and his participation for selecting the most relevant apps. We also would like to thank Isabell Wolhgennant who has laid down a solid basis for making this market study is possible. This project has been funded with support from the European Commission [Erasmus+ grant number 2018-1-LI01-KA203-000107]. This publication reflects the views only of the authors, and the Commission cannot be held responsible for any use which may be made of the information contained therein.

\section{References}

[1] H. Huygelier, B. Schraepen, R. van Ee, V. V. Abeele, and C. R. Gillebert, "Acceptance of immersive head-mounted virtual reality in older adults," Scientific reports, vol. 9, no. 1, pp. 1-12, 2019.

[2] I. Wohlgenannt, A. Simons, and S. Stieglitz, "Virtual reality," Business \& Information Systems Engineering, pp. 1-7, 2020.

[3] K. Amokrane and D. Lourdeaux, "Virtual reality contribution to training and risk prevention.," in $I C-A I$, pp. 726-732, 2009.

[4] Y. Zhou, S. Ji, T. Xu, and Z. Wang, "Promoting knowledge construction: a model for using virtual reality interaction to enhance learning," Procedia computer science, vol. 130, pp. 239-246, 2018.

[5] J. DeVaney, G. Shimshon, M. Rascoff, and J. Maggioncalda, "Higher ed needs a long-term plan for virtual learning," Harvard business review, May 2020.

[6] L. Calvet, P. Bourdin, and F. Prados, "Immersive technologies in higher education: Applications, challenges, and good practices," in Proc. 2019 3rd Int. Conf. on Education and E-Learning, pp. 95-99, 2019.

[7] N. M. Papachristos, I. Vrellis, and T. A. Mikropoulos, "A comparison between oculus rift and a low-cost smartphone vr headset: immersive user experience and learning," in IEEE 17th ICALT, pp. 477-481, IEEE, 2017.

[8] L. Freina and M. Ott, "A literature review on immersive virtual reality in education: state of the art and perspectives," in The int. scien. conf. elearning and software for education, pp. 10-1007, 2015.

[9] B. Chavez and S. Bayona, "Virtual reality in the learning process," in World Conference on Information Systems and Technologies, pp. 1345-1356, Springer, 2018.

[10] S. Kavanagh, A. Luxton-Reilly, B. Wuensche, and B. Plimmer, "A systematic review of virtual reality in education," Themes in Science and Technology Education, vol. 10, no. 2, pp. 85-119, 2017.

[11] I. Wohlgenannt, J. Fromm, S. Stieglitz, J. Radianti, and T. A. Majchrzak, "Virtual reality in higher education: Preliminary results from a design-science-research project," in Proc. 28th ISD, AIS Electronic Library (AISeL), 2019.

[12] J. Radianti, T. A. Majchrzak, J. Fromm, and I. Wohlgenannt, "A systematic review of immersive virtual reality applications for higher education: Design elements, lessons learned, and research agenda," Computers \& Education, vol. 147, p. 103778, 2020.

[13] C. Shuler, "ilearn: A content analysis of the itunes app store's education section," in Proc. 8th World Conf. on Mobile and Contextual Learning, p. 149, 2009.

[14] T. Hayes, "Mobile apps for 21st century skills: a quantitative analysis of educational mobile apps on graphite. org," in EdMedia+ Innovate Learning, pp. 1630-1637, AACE, 2016.

[15] E. Pechenkina, D. Laurence, G. Oates, D. Eldridge, and D. Hunter, "Using a gamified mobile app to increase student engagement, retention and academic achievement," International Journal of Educational Technology in Higher Education, vol. 14, no. 1, pp. 1-12, 2017.

[16] M. N. Callaghan and S. M. Reich, "Are educational preschool apps designed to teach? an analysis of the app market," Learning, Media and Technology, vol. 43, no. 3, pp. 280-293, 2018.

[17] I. Stojšić, A. I. Džigurski, O. Maričić, L. I. Bibić, and S. D. Vučković, "Possible application of virtual reality in geography teaching," Journal of Subject Didactics, vol. 1, no. 2, pp. 83-96, 2016.

[18] V. KARSLİ and M. B. KARSLİ, "Investigation of virtual reality applications as a tool in language learning," Takvim-i Vekayi, vol. 8, no. 1, pp. 80-95, 2020.

[19] N. R. Haddaway, A. M. Collins, D. Coughlin, and S. Kirk, "A rapid method to increase transparency and efficiency in web-based searches," Environmental Evidence, vol. 6, no. 1, p. 1, 2017.

[20] K. Higuchi, "Kh coder 3 reference manual," Kioto (Japan): Ritsumeikan University, 2016.

[21] J. Webster and R. T. Watson, "Analyzing the past to prepare for the future: Writing a literature review," MIS quarterly, pp. xiii-xxiii, 2002.

[22] V. E. Ekkelenkamp, A. D. Koch, R. A. de Man, and E. J. Kuipers, "Training and competence assessment in gi endoscopy: a systematic review," Gut, vol. 65 , no. 4, pp. 607-615, 2016.

[23] C. M. Harrington, D. O. Kavanagh, J. F. Quinlan, D. Ryan, P. Dicker, D. O'Keeffe, O. Traynor, and S. Tierney, "Development and evaluation of a trauma decision-making simulator in oculus virtual reality," The American Journal of Surgery, vol. 215, no. 1, pp. 42-47, 2018. 